\title{
Adsorção de cromo (VI) por carvão ativado granular de soluções diluídas utilizando um sistema batelada sob pH controlado
}

\author{
Renata Santos SOUZA', Samira Maria Leão CARVALHO², Márcio Ronald Lima GARCIA JÚNIORº , Rafael \\ Santos Fernandes SENA ${ }^{4}$
}

\begin{abstract}
RESUMO
$\mathrm{Na}$ Amazônia o cromo é empregado principalmente na indústria de couro e de madeira, sendo responsável por vários problemas de saúde porque é tóxico para os seres vivos. A remoção de cromo de efluentes industriais é feita por meio de diversos processos como a adsorção. Este trabalho mostra os resultados da adsorção de $\operatorname{Cr}(\mathrm{VI})$ por carvão ativado granular comercial (CAG) como adsorvente de soluções diluídas empregando um sistema de adsorção batelada com controle de $\mathrm{pH}$. Os grupos funcionais da superfície do CAG foram determinados pelo método de Boehm. Além disso, o efeito do $\mathrm{pH}$ na adsorção de $\mathrm{Cr}(\mathrm{VI})$, o equilíbrio e a cinética de adsorção foram estudados nas condiçóes experimentais $(\mathrm{pH}=6, \mathrm{MA}=6 \mathrm{~g}$, tempo de adsorção $90 \mathrm{~min}$.). Na superfície do CAG, os grupos carboxílicos foram determinados em maior concentração (MAS=0,43 mmol $/ \mathrm{gCAG}$ ), estes, presentes em concentrações elevadas aumentam a adsorção do metal, principalmente em valores de $\mathrm{pH}$ ácidos. A capacidade de adsorção é dependente do $\mathrm{pH}$ da solução, devido a sua influência nas propriedades de superfície do CAG e nas diferentes formas iônicas das soluções de $\mathrm{Cr}(\mathrm{VI})$. Os dados de equilíbrio da adsorção foram ajustados satisfatoriamente pela isoterma de Langmuir $\left(\mathrm{R}^{2}=0,988\right)$, tipo favorável. A partir da cinética de adsorção a $5 \mathrm{mg} / \mathrm{L}$ e $20 \mathrm{mg} / \mathrm{L}$, os resultados obtidos foram compatíveis com o valor limite preconizado na legislação nacional (Res. $\mathrm{n}^{\circ}$ 357/05). Portanto, para o sistema experimental utilizando CAG foi eficiente na remoção de $\mathrm{Cr}(\mathrm{VI})$ a partir de correntes líquidas contendo baixas concentraçôes do metal.
\end{abstract}

PALAVRas-chaVE: Adsorção, Cr(VI), CAG.

\section{Chromium (VI) adsorption by GAC from diluted solutions in batch system and controlled ph}

\begin{abstract}
In Amazonia, chromium is mainly used in the leather and wood industries. It is responsible for many health problems, because of its toxicity. These industries remove chromium waste by various processes, such as adsorption. This work shows the results of $\mathrm{Cr}(\mathrm{VI})$ adsorption by commercial granular activated carbon (GAC) as adsorbent from diluted solutions, and batch systems with controlled $\mathrm{pH}$. The functional groups on the CAG surface was carried out by the Boehm method. In addition, effect of $\mathrm{pH}$ on the $\mathrm{Cr}(\mathrm{VI})$ adsorption, adsorption equilibrium, and kinetic were studied under experimental conditions $(\mathrm{pH}$ $=6, \mathrm{MA}=6 \mathrm{~g}$, for $90 \mathrm{~min}$.). On the GAC surface, carboxylic groups were found to be in higher concentrations (MAS=0,43 $\mathrm{mmol} / \mathrm{gCAG})$, which increase the $\mathrm{Cr}(\mathrm{VI})$ adsorption, principally in acidic $\mathrm{pH}$ values. The adsorption capacity is dependent on the $\mathrm{pH}$ of the solution, due to its influence on the surface properties of the CAG and different ionic forms of the $\mathrm{Cr}(\mathrm{VI})$ solutions. The adsorption equilibrium data was adjusted satisfactorily by the Langmuir isotherm $\left(\mathrm{R}^{2}=0,988\right)$, favorable type. From kinetics adsorption of $20 \mathrm{mg} / \mathrm{L}$ and $5 \mathrm{mg} / \mathrm{L}$, the results were compatible with the national legislation (Res. $\mathrm{n}^{\circ} 357 / 05$ ). Therefore, the experimental system using (CAG) was efficient in removing the $\mathrm{Cr}(\mathrm{VI})$ from liquid streams containing low concentrations of the metal
\end{abstract}

KEY WORDS: Adsorption, Chromium (VI), GAC.

\footnotetext{
1 Universidade Federal do Pará, Programa de Pós-graduação em Engenharia Química. Conjunto Bela Vista, Travessa Macapá 176, Val de Cães, 66617250, Belém- Pará- Brasil. E-mail: rena.ssouza@gmail.com

2 Universidade Federal do Pará, Programa de Pós-graduação em Engenharia Química. E-mail: sleao@ufpa.br

3 Universidade Federal do Pará, Faculdade de Engenharia Química. E-mail: marcio_garcia13@hotmail.com

${ }^{4}$ Universidade Federal do Pará, Faculdade de Engenharia Química. E-mail: fernandeseq2006@yahoo.com.br
} 


\section{INTRODUÇÃO}

Atualmente na Amazônia muitos são os poluentes constituídos por cromo hexavalente $(\mathrm{Cr}(\mathrm{VI}))$, na fabricação de aços inoxidáveis e outras ligas metálicas, na indústria de refratários para fazer tijolos de fornos metalúrgicos, na indústria de galvanoplastia, manufatura de pigmentos, e na região, principalmente, nos curtumes e tratamento de madeira (Giannetti et al., 2007).

A presença de $\mathrm{Cr}(\mathrm{VI})$ em rios e lagos é responsável por vários problemas de saúde de animais, plantas e seres humanos porque este metal é biocumulativo e tóxico para os seres vivos (Costodes et al., 2003).

A hidrólise de $\mathrm{Cr}(\mathrm{VI})$ produz predominantemente as espécies cromato $\left(\mathrm{CrO}_{4}^{2}\right)$, cromato ácido $\left(\mathrm{HCrO}_{4}^{-}\right)$e dicromato $\left(\mathrm{Cr}_{2} \mathrm{O}_{7}^{-2}\right)$ dependendo do $\mathrm{pH}$ e da concentração de $\mathrm{Cr}(\mathrm{VI}) \mathrm{em}$ solução (Mohana e Pittman, 2006).

Segundo Liu et al.(2007), vários métodos de tratamento de efluentes líquidos contaminados por compostos de $\mathrm{Cr}(\mathrm{VI})$ foram estudados, dentre eles a adsorção se mostrou efetiva e por isso é bastante utilizada, principalmente na remoção de compostos-traços orgânicos e inorgânicos de grandes volumes de efluentes líquidos e gasosos, isto é, nos processos de polimento de efluentes; e sua eficiência está diretamente relacionada com o tipo de adsorvente utilizado. Além disso, é um processo de controle da poluição capaz de adequar as correntes de efluentes para o lançamento ou disposição final, de acordo com os limites estabelecidos na legislação brasileira, resolução n $357 / 05$ do Conselho Nacional de Meio Ambiente (Brasil, 2005), cujo valor máximo permitido de cromo total é de $0,5 \mathrm{mg} / \mathrm{L}$.

Dentre os adsorvestes, o carvão ativado granular (CAG), é um dos mais utilizados. A eficiência de processo, com a utilização de CAG, depende não somente das propriedades físicas do adsorvente, mas também das suas condições de ativação (Hamadi et al., 2001).

Grande parte das publicações sobre adsorção de $\mathrm{Cr}(\mathrm{VI})$ por CAG investiga soluçôes de concentrações do metal (superior a $100 \mathrm{mg} / \mathrm{L}$ ). Neste trabalho foram estudadas soluções diluídas na faixa de concentração entre $5 \mathrm{mg} / \mathrm{L}$ e $25 \mathrm{mg} / \mathrm{L}$, conforme citado na literatura: $5 \mathrm{mg} / \mathrm{L}$ e $50 \mathrm{mg} / \mathrm{L}$ (Liu et al., 2007), $10 \mathrm{mg} / \mathrm{L}$ e $30 \mathrm{mg} / \mathrm{L}$ (Mohanty et al., 2005), $5 \mathrm{mg} / \mathrm{L}$ e $25 \mathrm{mg} / \mathrm{L}$ (Babel e Kurniawan, 2004), 5mg/L e 25mg/L (Selvi et al., 2001). Nestes estudos, o controle do $\mathrm{pH}$ é determinante para a remoção de $\mathrm{Cr}(\mathrm{VI})$ pelo adsorvente, uma vez que, esse parâmetro afeta as cargas da superfície do adsorvente (Natale, 2007), e determinar as formas iônicas do metal em solução (Hamadi et al.,2001).

O principal objetivo deste trabalho foi avaliar a capacidade de adsorção de carvão ativado granular comercial (CAG) na remoção de $\mathrm{Cr}(\mathrm{VI})$ a partir de soluções diluídas, empregando um sistema de adsorção batelada, com controle de $\mathrm{pH}$.

\section{MATERIAIS E MÉTODOS}

\section{SISTEMA EXPERIMENTAL}

Nos ensaios de adsorção de $\mathrm{Cr}(\mathrm{VI})$ por CAG foi empregado o sistema experimental (Figura 1) constituído de frasco cônico $(250 \mathrm{~mL})$ contendo a solução teste e o adsorvente (CAG). Ao frasco cônico foram acoplados um $\mathrm{pH}$-metro digital (Schott) (para o controle da medida em tempo real) e uma bomba dosadora (Masterflex) de solução (ácida ou básica) para o controle do $\mathrm{pH}$. A agitação foi realizada em uma mesa agitadora (B. Braun Biotech) com controladores de velocidade e tempo. Os ensaios foram realizados a temperatura ambiente $\left(26^{\circ} \mathrm{C}\right)$.

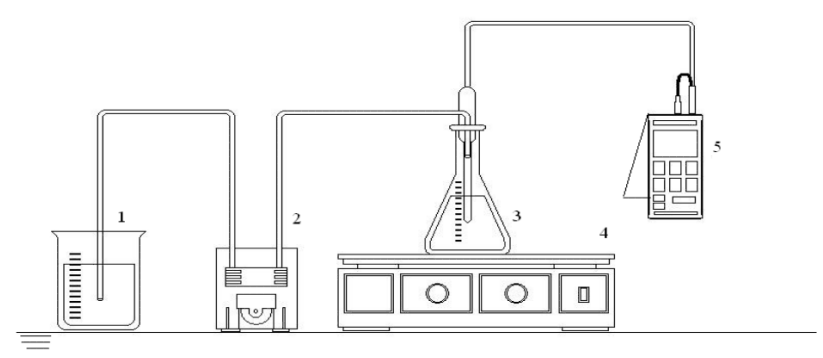

1 - Recipiente de Alimentação, 2 - Bomba Dosadora, 3 - Solução + CAG, 4 - Mesa Agitadora, 5 - pHmetro Digital

Figura 1 - Sistema Experimental em Batelada com controle de pH.

\section{PREPARAÇÃO DO ADSORVENTE}

Carvão ativado granular comercial (CAG) foi utilizado como adsorvente, com as seguintes propriedades físicas: área superficial (BET) de $754\left(\mathrm{~m}^{2} / \mathrm{g}\right)$; densidade aparente de $0,5 \mathrm{~g} /$ $\mathrm{mL}$; diâmetro médio de Sauter de 2,4 (mm). Previamente, a sua utilização o CAG foi submetido aos seguintes procedimentos: enxágüe em água deionizada, fervura durante três horas em água deionizada, secagem em estufa a $120^{\circ} \mathrm{C}$.

\section{ENSAIOS DE ADSORÇÃOO}

Soluções de $\mathrm{Cr}(\mathrm{VI})$ nas concentrações 5 e $20 \mathrm{mg} / \mathrm{L}$ (em $\mathrm{Cr}(\mathrm{VI})$ ) foram preparadas apartir de dicromato de potássio $\left(\mathrm{K}_{2} \mathrm{Cr}_{2} \mathrm{O}_{7}\right) 50 \mathrm{mg} / \mathrm{L}$, obtida conforme metodologia padrão (APHA, 1995). Após a adsorção a concentração de cromo residual na solução foi determinada, pelo método colorimétrico (APHA, 1995) empregando-se 1,5 difenilcarbazida, em $540 \mathrm{~nm}$ por espectrofotometria e por espectrometria de absorção atômica (APHA, 1995). A remoção percentual de $\mathrm{Cr}(\mathrm{VI})$ foi calculada empregando-se a Equação 1. 


$$
R(\%)=\frac{\left(C_{i}-C_{e}\right)}{C_{i}} \times 100
$$

Onde: $C_{i}$ e $C_{e}$, são respectivamente as concentrações inicial e de equilíbrio de $\mathrm{Cr}(\mathrm{VI})$ em solução (mg/L).

\section{GRUPOS FUNCIONAIS PRESENTES NA SUPERFÍCIE DO CAG}

A caracterização dos grupos funcionais da superfície do CAG foi realizada segundo a metodologia de Boehm (Bueno;Carvalho, 2007). A titulação de Boehm identifica os grupos funcionais ácidos presentes na superfície de carvões ativados, como grupos carboxílicos (-COOH), hidroxil fenólicos (-OH) e lactonas (-COOR). É aceito que o hidróxido de sódio $(\mathrm{NaOH})$ neutraliza os grupos carboxílicos, lactonas e fenólicos, por sua vez o carbonato de sódio $\left(\mathrm{Na}_{2} \mathrm{CO}_{3}\right)$ neutraliza grupos carboxílicos e lactonas e o bicarbonato de potássio $\left(\mathrm{KHCO}_{3}\right)$ neutraliza somente grupos carboxílicos (Schneider et al., 2005; Zhao et al., 2005; Bueno e Carvalho, 2007). Na aplicação da metodologia 2,0g de CAG foram colocados em contato com $20 \mathrm{~mL}$ de soluções de hidróxido de sódio $(\mathrm{NaOH})$ 0,1 mol/L, bicarbonato de potássio $\left(\mathrm{KHCO}_{3}\right)$ 0,05 mol/L e carbonato de sódio $\left(\mathrm{Na}_{2} \mathrm{CO}_{3}\right)$ $0,05 \mathrm{~mol} / \mathrm{L}$, em erlenmeyers de $250 \mathrm{~mL}$. Os frascos com as soluções e o adsorvente foram fechados e agitados por 24 horas, em mesa agitadora a $120 \mathrm{rpm}$. Posteriormente, as soluçóes foram filtradas e tituladas com solução de ácido clorídrico $(\mathrm{HCl} 0,1 \mathrm{~mol} / \mathrm{L})$. Os grupos funcionais identificados no CAG foram calculados, em $\mathrm{mmol} / \mathrm{g}(M A S)$, pela Equação 2 (Bueno e Carvalho, 2007).

$$
M A S=\frac{0,1 \cdot f \cdot(T a-T) \cdot\left(\frac{50}{20}\right)}{W}
$$

Onde: $f$, fator de pureza do ácido; Ta o volume de ácido consumido pela base após o contato com o carvão granular na titulação (em $\mathrm{mL}) ; T$, volume de ácido consumido pela base em branco (em mL) e $W$, massa de carvão utilizada (em gramas);

\section{ESTUDO DO EFEITO DO PH NA REMOÇÃO DE CR(VI) POR CAG}

Os valores de $\mathrm{pH}$ da solução de $\mathrm{Cr}(\mathrm{VI})$ estudados foram 3; $4 ; 5 ; 5,5 ; 6 ; 6,5 ; 7 ;$ e 8 . Para cada ensaio foi utilizado $100 \mathrm{~mL}$ da solução de $\mathrm{Cr}(\mathrm{VI})$ de $20 \mathrm{mg} / \mathrm{L}$, massa de adsorvente (MA) de $6,0 \mathrm{~g}$ e tempo de contato de 90 minutos. O pH da solução (Cr(VI)/CAG) foi ajustado e controlado (HCL $0,1 \mathrm{~mol} / \mathrm{L}$ ou $\mathrm{NaOH} 0,1 \mathrm{~mol} / \mathrm{L}$ ) durante a realização de todos os ensaios. A concentração de $\mathrm{Cr}(\mathrm{VI})$ no filtrado foi determinada por espectrometria de absorção atômica. Os valores de concentração e massa de adsorvente foram escolhidos com base em dados apresentados em trabalhos anteriores (Souza et al., 2007).

\section{EQUILÍBRIO DE ADSORÇÃO}

As seguintes condições experimentais foram utilizadas: $p H=6,0 ; M A=6,0 \mathrm{~g}$, velocidade de agitação $120 \mathrm{rpm}$, tempo de contato 90 minutos e temperatura ambiente $\left(26^{\circ} \mathrm{C}\right)$. Para cada ensaio $100 \mathrm{~mL}$ da solução de $\mathrm{Cr}(\mathrm{VI})$ (concentração inicial da solução entre $5 \mathrm{mg} / \mathrm{L}$ e $20 \mathrm{mg} / \mathrm{L}$, foram colocados em contato com o CAG. A solução foi submetida à agitação e o valor do $p H$ foi mantido constante, com a adição de solução de ácido

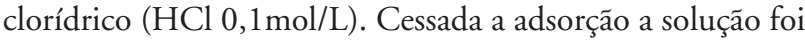
filtrada concentração de $\mathrm{Cr}(\mathrm{VI})$ na solução foi determinada por espectrofotometria.

A isoterma de Langmuir linearizada foi utilizada como modelo matemático para correlacionar os dados experimentais. Segundo a literatura consultada, é o modelo matemático mais utilizado para estudos de equilíbrio de adsorção do sistema Cr(VI)/Carvão Ativado (Bajpai et al., 2000; Ozacar, 2003), além de ser aplicada com sucesso em inúmeros processos de adsorção. A isoterma de Langmuir linearizada é representada pela Equação 3.

$$
\frac{1}{q_{e}}=\frac{1}{q_{o}}+\left(\frac{1}{K_{L}}\right) \frac{1}{C_{e}}
$$

Onde: $q_{e}$, quantidade adsorvida na fase sólida $[\mathrm{mg} \mathrm{Cr}$ (VI)/g CAG]; $C_{e}$, concentração de $\mathrm{Cr}(\mathrm{VI})$ em equilíbrio na fase líquida $(\mathrm{mg} / \mathrm{L}) ; q_{0}[\mathrm{mg} \mathrm{Cr}(\mathrm{VI}) / \mathrm{g} \mathrm{CAG}]$ e $b(\mathrm{~L} / \mathrm{g})$ são as constantes de Langmuir; $q_{o}$, máxima capacidade de cobertura da monocamada e $b$ está relacionado com a energia de adsorção. O parâmetro $K_{L}=\mathrm{q}_{0}$. b é denominado constante de equilíbrio de Langmuir.

De acordo com Kadirvelu et al., (2001), uma característica essencial da isoterma de Langmuir pode ser explicada em termos do parâmetro $\left(R_{L}\right)$ denominado fator de separação, definido pela Equação 4, que possibilita avaliar a forma da isoterma: isoterma reversivel $\left(R_{L}=0\right)$; favorável $\left(0<R_{L}<1\right)$; linear $\left(R_{L}=1\right)$; desfavorável $\left(R_{L}>1\right) . R_{L}$ indica a eficiência do adsorvente sob estudo.

$$
R_{L}=\frac{1}{1+b \cdot C_{i}}
$$

Onde: $b$, constante de Langmuir; $C$, concentração inicial do metal em solução $(\mathrm{mg} / \mathrm{L})$.

A massa de $\mathrm{Cr}(\mathrm{VI})$ adsorvida no CAG foi calculada empregando-se o balanço de massa, conforme a Equação 5. 


$$
q_{e}=\frac{\left(C_{i}-C_{e}\right) \cdot V}{W}
$$

Onde: $q$, quantidade de metal adsorvida na fase sólida [mg Cr(VI)/g CAG]; $V$, volume de solução (L); $W$, massa de adsorvente $(\mathrm{g}) ; C_{i}$ e $C_{e}$, são as concentraçôes inicial e de equilíbrio de $\mathrm{Cr}(\mathrm{VI})$ em solução $(\mathrm{mg} / \mathrm{L})$, respectivamente.

\section{ENSAIOS DE CINÉTICA DE ADSORÇÃO}

As seguintes condiçōes experimentais foram utilizadas: $p H=6,0, M A=6,0 \mathrm{~g}$, velocidade de agitação $120 \mathrm{rpm}, C_{i}=$ $5 \mathrm{mg} / \mathrm{L}$ ( 10 ensaios) e $C_{i}=20 \mathrm{mg} / \mathrm{L}$ ( 10 ensaios) em diferentes intervalos de tempo (tempo total de adsorção $90 \mathrm{~min}$ ). As amostras foram preparadas empregando-se $100 \mathrm{~mL}$ de solução de $\operatorname{Cr}(\mathrm{VI})\left(C_{i}=5 \mathrm{mg} / \mathrm{L}\right.$ ou $\left.C_{i}=20 \mathrm{mg} / \mathrm{L}\right)$ e $6,0 \mathrm{~g}$ de CAG. O pH da solução foi ajustado $(p H=6,0)$ e a solução (teste) foi submetida à agitação durante $5,10,15,20,30$, 40, 50, 75 e $90 \mathrm{~min}$. Nos ensaios empregando concentração inicial de $20 \mathrm{mg} / \mathrm{L}$ os tempos de adsorção foram de $2,4,6$, $10,20,30,45,60,75$ e $90 \mathrm{~min}$. $\mathrm{O} \mathrm{pH}$ das amostras foram mantidos constantes durante a realização dos ensaios. A remoção percentual de $\mathrm{Cr}(\mathrm{VI})$ foi calculada empregando-se a Equação 1.

\section{RESULTADOS E DISCUSSÃO}

\section{GRUPOS FUNCIONAIS PRESENTES NA SUPERFÍCIE DO CAG}

Os resultados da titulação Boehm são mostrados na Tabela 1.

Tabela 1 - Resultados da titulação de Boehm.

\begin{tabular}{lc} 
Grupos funcionais & $\begin{array}{c}\mathrm{MAS} \\
(\mathrm{mmol} / \mathrm{g} \mathrm{CAG})\end{array}$ \\
\hline$-\mathrm{COOH}$ & 0,43 \\
$-\mathrm{OH}$ & 0,33 \\
$-\mathrm{COOR}$ & 0,01 \\
\hline
\end{tabular}

No CAG estudado, os grupos ácidos carboxílicos foram encontrados em maior quantidade ( $M A S=0,43 \mathrm{mmol} / \mathrm{gCAG}$ ), em comparação com os grupos hidroxil fenólicos e grupos lactonas (Tabela 1). Estes dois últimos grupos são considerados relativamente fracos e se dissociam em valores de $\mathrm{pH}$ mais altos que os grupos ácidos carboxílicos. Estes, quando presentes em concentrações elevadas, em comparação com os outros grupos funcionais melhoram o processo de adsorção, principalmente se o $\mathrm{pH}$ de trabalho estiver situado na faixa ácida, que favorece a protonação desses grupos (Liu et al., 2007).

\section{EFEITO DO PH NA REMOÇÃO DE CR(VI) POR CAG}

A Figura 2 mostra o efeito do $p H$ na remoção percentual de $\mathrm{Cr}(\mathrm{VI})$ por CAG.

$\mathrm{Na}$ Figura 2 é observado que a remoção percentual do metal (\%R) tem um pequeno aumento com o aumento do $p H$ na faixa entre 3,0 a 6,0 elevando-se de $94 \%$ até $98 \%$ ao atingir $p H=6,0$; diminuindo em seguida até $16 \%$ em $p H=8,0$.

Os resultados obtidos, mostrados na Figura 2, referem-se à determinação de $\mathrm{Cr}(\mathrm{VI})$ por espectrometria de absorção atômica. Esses resultados mostram que a capacidade de adsorção é dependente do $\mathrm{pH}$ da solução. $\mathrm{O}$ pH determina a extensão da remoção de $\mathrm{Cr}(\mathrm{VI})$, pois promove a formação de cargas de superfície favoráveis à adsorção do metal e determina as formas iônicas do $\mathrm{Cr}(\mathrm{VI})$ em solução (Hamadi et al., 2001). A diminuição na remoção percentual do metal, em valores de $\mathrm{pH}$ superiores a $p H=6,0$, pode ser atribuída a uma redução significativa da concentração de cargas positivas da superfície do adsorvente (SELVI et al., 2001) pela desprotonação de grupos ácidos e a competitividade entre as espécies de cromo $\left(\mathrm{HCrO}^{-}\right.$ $\left.{ }_{4} \mathrm{CrO}_{4}^{-2}\right)$ e os íons $\mathrm{OH}$, em solução, para a adsorção nos sítios do adsorvente. Em meios ácidos, ocorre o aumento de cargas positivas na superfície do adsorvente que favorecem a atração eletrostática entre os grupos de superfície e a espécie do metal presente em solução ( $\mathrm{HCrO}_{4}^{-}, \mathrm{CrO}_{4}^{-2}, \mathrm{Cr}_{2} \mathrm{O}_{7}^{-2}$ ) (Selvi et al., 200; GAO et al.,2002; Arslan e Pehlivan, 2007;).

Em soluções diluídas de $\mathrm{Cr}(\mathrm{VI})$ em $p H$ igual a 1,0 íons cromo existem na forma de $\mathrm{H}_{2} \mathrm{CrO}_{4}$ (ácido crômico) enquanto no intervalo de $\mathrm{pH}$ entre 1,0 e 6,0 coexistem diferentes formas de íons cromo tais como: $\mathrm{HCrO}_{4}^{-}$(íon cromato ácido), $\mathrm{Cr}_{2} \mathrm{O}_{7}^{-2}$, $\mathrm{Cr}_{3} \mathrm{O}_{10}, \mathrm{Cr}_{4} \mathrm{O}_{13}$, os íons $\mathrm{HCrO}_{4}^{-}$predominam em $\mathrm{pH}$ ácido; conforme o aumento do $\mathrm{pH}$ da soluçáo, os íons $\mathrm{HCrO}_{4}^{-}$se convertem em íons $\mathrm{CrO}_{4}^{-2}$ e íons, $\mathrm{Cr}_{2} \mathrm{O}_{7}^{-2}$ no intervalo de $\mathrm{pH}$ entre 1,0 e 6,0 . O equilíbrio existente entre as diferentes

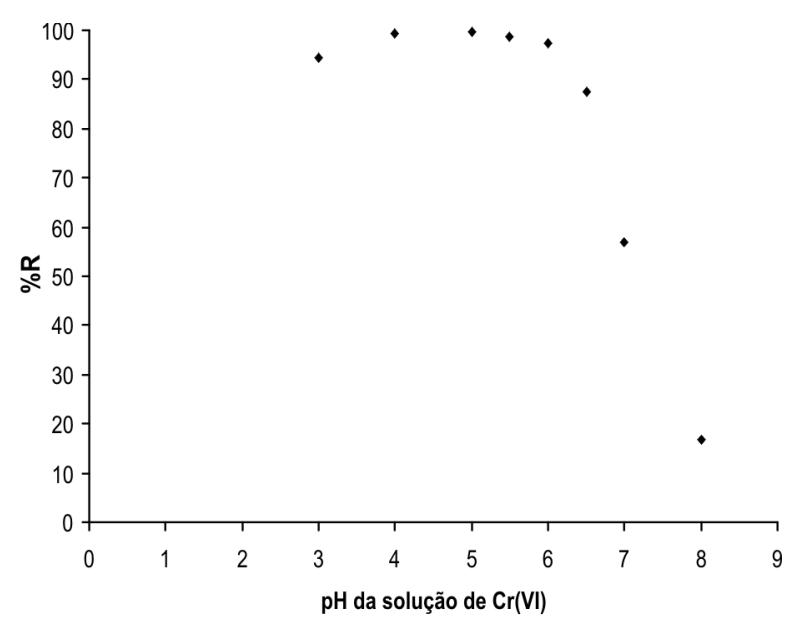

Figura 2 - Efeito do pH na remoção percentual de $\mathrm{Cr}$ (VI) por CAG. 
espécies iônicas de cromo são mostrados nas Equaçóes 6, 7 e 8 (Karthikeyan et al., 2005).

$$
\begin{array}{ll}
\mathrm{H}_{2} \mathrm{CrO}_{4} \leftrightarrows & \mathrm{H}^{+} \mathrm{HCrO}_{4}^{-} \\
\mathrm{HCrO}_{4}^{-} \leftrightarrows & \mathrm{H}^{+}+\mathrm{CrO}_{4}^{-} \\
2 \mathrm{HCrO}_{4}^{-} \leftrightarrows & \mathrm{Cr}_{2} \mathrm{O}_{7}^{-2}
\end{array}
$$

O estudo de influência do parâmetro pH na adsorção é importante para a decisão à cerca dos valores de $\mathrm{pH}$ a serem utilizados na otimização dos sistemas de adsorção, particularmente para adsorção em fase líquida, uma vez que, nesses sistemas tanto a especiação do metal como as cargas de superfície do adsorvente dependem do valor do $\mathrm{pH}$ (Babel;Kurniawan, 2004). A eficiência de remoção do metal por adsorção, comportamento observado na Figura 1, está relacionado ao par adsorbato/adsorvente e das condiçóes experimentais do sistema, mesmo um sistema similar constituído de $\mathrm{Cr}(\mathrm{VI}) / \mathrm{CAG}$, pode apresentar comportamento diferente dos resultados obtidos neste trabalho.

\section{EQUILÍBRIO DE ADSORÇÃO DE CROMO (VI) POR CAG}

Os resultados experimentais obtidos da adsorção de $\mathrm{Cr}$ (VI) por CAG, a partir das soluçōes com diferentes concentrações do metal, correlacionados à isoterma de Langmuir linearizada, são mostrados na Figura 3. Os parâmetros da isoterma de Langmuir, bem como, os valores do coeficiente de regressão, foram obtidos utilizando software estatístico (método dos mínimos quadrados), são mostrados na Tabela 2.

Conforme pode ser observado na Figura 3, a isoterma de Langmuir ajustou satisfatoriamente os dados experimentais da adsorção $\left(\mathrm{R}^{2}=0,988\right)$. $\mathrm{O}$ valor de $R_{L}$ calculado $\left(R_{L} \cong 0,115\right)$, situou-se entre 0 e 1 , que indica adsorção do tipo favorável.

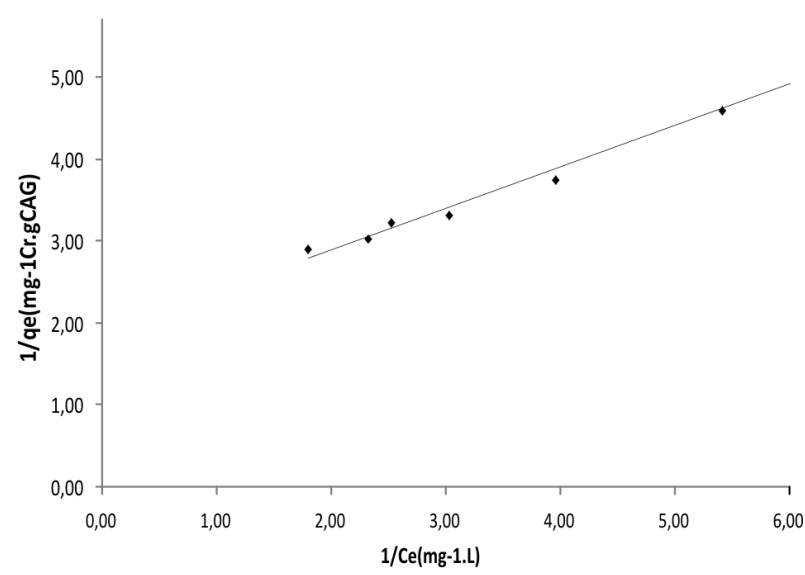

Figura 3 - Dados experimentais (pontos) ajustados pelo modelo da isoterma de Langmuir (linha).
Tabela 2 - Parâmetros da isoterma de Langmuir para a adsorção de $\mathrm{Cr}$ (VI)/CAG $\left(\mathrm{R}^{2}=0,988\right)$.

\begin{tabular}{ll}
\hline Qo $(\mathrm{mg} \mathrm{Cr} \mathrm{Vl} / \mathrm{g} \mathrm{CAG})$ & 0,54 \\
\hline$K_{L}(\mathrm{~L} / \mathrm{mg})$ & 1,96 \\
$\mathrm{~B}$ & 3,65 \\
\hline
\end{tabular}

A máxima capacidade de adsorção do $\mathrm{CAG}$, nas condições experimentais estudadas, foi de 0,54 ( $\mathrm{mgCrVI} / \mathrm{gCAG})$.

\section{ENSAIOS DE CINÉTICA DE ADSORÇÃO}

Os resultados de remoção percentual de Cr (VI) $(\% R$ ) obtidos a partir dos ensaios da cinética de adsorção, para $C_{i}=5 \mathrm{mg} / \mathrm{L}$ e $C_{i}=20 \mathrm{mg} / \mathrm{L}$ são mostrados nas Figuras 4 e 5 , respectivamente.

Conforme a Figura 4, após 30 min. de adsorção, 73\% da capacidade do CAG foi atingida. O sistema atingiu o

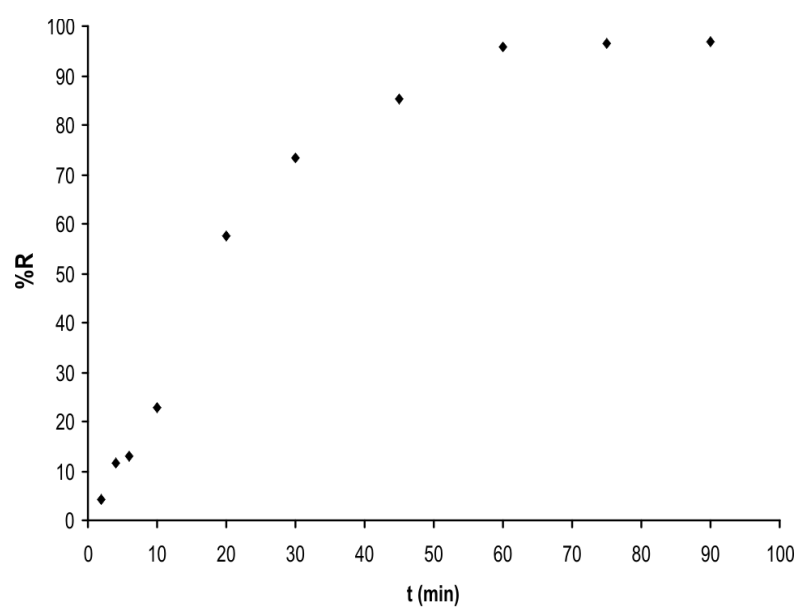

Figura 4 - Dados experimentais de cinética de adsorção de $\mathrm{Cr}(\mathrm{VI})$ por CAG, $C_{i}=5 \mathrm{mg} / \mathrm{L}, \mathrm{pH}=6,0$.

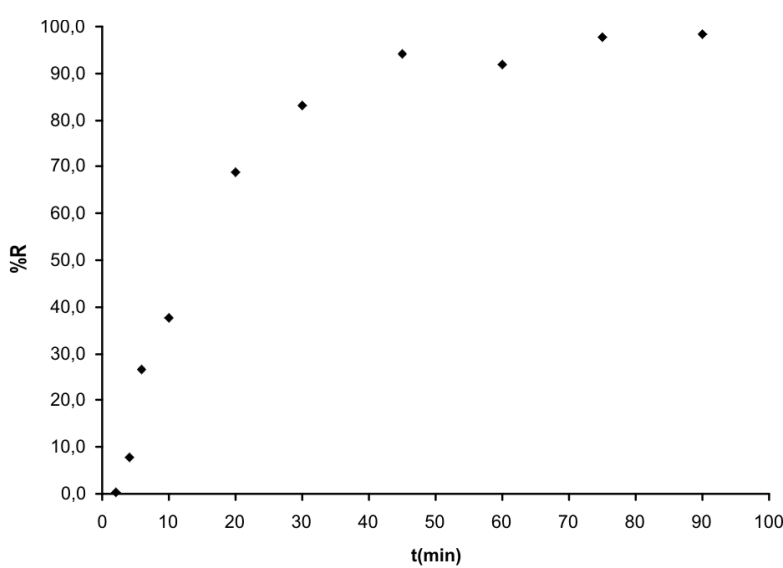

Figura 5 - Dados experimentais de cinética de adsorção de $\mathrm{Cr}$ (VI) por CAG $C_{i}=20 \mathrm{mg} / \mathrm{L}, p H=6,0$ 
equilíbrio após 60 minutos de processo. Ao final da adsorção (90 min) obteve-se remoção de $97 \%$ do metal e concentração de equilíbrio $(C)$ (na solução) igual a $0,2 \mathrm{mg} / \mathrm{L}$ (Tabela 3 ).

A partir da Figura 5, observa-se que a remoção de $\mathrm{Cr}(\mathrm{VI})$ foi de aproximadamente $99 \%$ após 90 min de processo e concentração de equilíbrio $\left(C_{e}\right)$ foi igual a $0,3 \mathrm{mg} / \mathrm{L}$ (Tabela 3).

As Tabelas 3 e 4 mostram os valores da concentração de equilíbrio do metal $\left(C_{e}\right)$ para a cinética de adsorção, concentrações iniciais, respectivamente $C_{i}=5 \mathrm{mg} / \mathrm{L}$ e $C_{i}$ $=20 \mathrm{mg} / \mathrm{L}$.

Os valores de concentração de equilíbrio $\left(C_{e}\right)$ do metal em solução obtidos, conforme mostrado nas Tabelas 3 e 4, após $60 \mathrm{~min}$ de adsorção para a $C_{i}=5 \mathrm{mg} / \mathrm{L}$; e após $75 \mathrm{~min}$ de adsorção para $C_{i}=20 \mathrm{mg} / \mathrm{L}$, são compatíveis com o valor limite preconizado na legislação nacional (Res. no 357/05) mostrando que a partir do sistema experimental utilizado e nas condições estudadas, carvão ativado granular comercial (CAG) foi eficiente na remoção de $\mathrm{Cr}(\mathrm{VI})$, a partir de correntes líquidas contendo baixas concentrações do metal.

Tabela 3 - Concentrações de equilíbrio $\left(C_{e}\right)$ para a cinética de adsorção $\left(C_{i}\right.$ $=5,0 \mathrm{mg} / \mathrm{L})$

\begin{tabular}{lll}
\hline$t(\min )$ & $C_{\mathrm{e}}(\mathrm{mg} / \mathrm{L})$ & $\% \mathrm{R}$ \\
\hline 2 & 5,6 & 4 \\
4 & 5,2 & 12 \\
6 & 5,1 & 13 \\
10 & 4,5 & 23 \\
20 & 2,5 & 58 \\
30 & 1,6 & 73 \\
45 & 0,8 & 85 \\
60 & 0,3 & 96 \\
75 & 0,2 & 96 \\
90 & 0,2 & 97 \\
\hline
\end{tabular}

Tabela 4 - Concentrações de equilíbrio (Ce) para a cinética de adsorção $(C$ $=20,0 \mathrm{mg} / \mathrm{L})$.

\begin{tabular}{lll}
\hline$T(\min )$ & $C_{e}(\mathrm{mg} / \mathrm{L})$ & $\% R$ \\
\hline 2 & 20,8 & $<1$ \\
4 & 19,3 & 8 \\
6 & 15,3 & 27 \\
10 & 13,0 & 38 \\
20 & 6,5 & 69 \\
30 & 3,5 & 83 \\
45 & 1,2 & 94 \\
60 & 1,7 & 92 \\
75 & 0,5 & 98 \\
90 & 0,3 & 99 \\
\hline
\end{tabular}

\section{CONCLUSÕES}

$\mathrm{O}$ estudo do efeito do $\mathrm{pH}$ na eficiência de remoção de $\mathrm{Cr}(\mathrm{VI})$ mostrou valores superiores a $90 \%$, no intervalo de $\mathrm{pH}$ (entre 3,0 e 6,0).

No sistema de adsorção em batelada, o controle de $\mathrm{pH}$ foi de fundamental importância, na eficiência de remoção do metal. Contudo, o controle foi necessário, em tempo real, durante todo o tempo de contato.

Os dados do estudo de equilíbrio de adsorção foram ajustados, satisfatoriamente pela isoterma de Langmuir, o valor de $\mathrm{R}_{\mathrm{L}}$ calculado indicou adsorção do tipo favorável.

Os resultados de remoção percentual de $\mathrm{Cr}(\mathrm{VI})(\% R)$ obtidos a partir dos ensaios da cinética de adsorção em sistema batelada, mostraram que a concentração de equilíbrio $(C)$ do metal na solução, após adsorção, são compatíveis com o valor limite (cromo total) preconizados na legislação Nacional vigente, com relaçáo ao lançamento de efluentes líquidos contaminados por cromo.

Então, considerando as etapas executadas neste trabalho, nas condiçôes experimentais descritas, o sistema de adsorção $\mathrm{Cr}(\mathrm{VI}) / \mathrm{CAG}$ em batelada foi eficiente na remoção de $\mathrm{Cr}(\mathrm{VI})$ em todas as combinações de variáveis de processo $(p H, M A$ e $C_{i}$ ), porém sob $\mathrm{pH}$ controlado durante todo o processo de adsorção.

\section{BIBLIOGRAFIA CITADA}

Abdessemed, D.; Nezzal, G. 2002. Treatment of Primary Effluent by Coagulation-Adsorption-Ultrafiltration for Reuse. Desalination, 152: 367-373.

American Public Health Association (APHA). (1995). Standard Methods for the Examination of Water and Wastewater, 19th edition, Washington. EUA.

Arslan, G.; Pehlivan, E. 2007. Batch removal of chromium(VI) from aqueous solution by Turkish brown coals. Bioresource Technology, (98): 2836-2845.

Babel, S.; Kurniawan, T A. 2004. Cr(VI) removal from synthetic wastewater using coconut shell charcoal and commercial activated carbon modified with oxidizing agents and/or chitosan. Chemosphere, 54: 951-967.

Bajpai, A.K; Rajpoot, M.; Mishra, D.D. 2000. Static and Kinetic Studies on the Adsorption Behavior of Sulfadiazene. Adsorption, 6: 349-357.

Brasil, 2005. Resolução do Conselho Nacional do Meio Ambiente (CONAMA), número 357, publicada em 17 de março de 2005. Diário Oficial da República Federativa do Brasil, Brasília, DF.

Bueno, C.I.C.; Carvalho, W.A. 2007. Remoção de Chumbo (II) em sistemas descontínuos por carvões ativados com ácido fosfórico e com vapor. Quim. Nova.30(08): 1911-1918.

Costodes, V.C.T; Fauduet, H.; Porte, C; Delacroix, A. 2003. Removal of $\mathrm{Cd}(\mathrm{II})$ and $\mathrm{Pb}$ (II) ions, from aqueous solutions, by adsorption onto sawdust of Pinus sylvestris. Jornal of Hazardous Materials, 105: 121-142. 
Guo, Y.; Qi, J.; Yang, S.; Yu, K,; Wang, Z.; Xu, H. 2002. Adsorption of $\mathrm{Cr}$ (VI) on micro and mesoporous rice husk-based active carbon. Materials Chemistry and physics, 78: 132-137.

Giannetti, B.F.; Almeida, C.M.V.B.; Bonilla, S.H.; Vendrameto, O. Nosso Cromo de Cada Dia: Benefícios e Riscos. [S. 1.: s. n.]. Disponível em: <http://www.hottopos.com.br/regeq8/biaggio. htm> . Acesso em 20/01/ 2007.

B.F. Giannetti,

Hamadi, N.K.; Chen, X.D.; Farid, M.M.; Lu, M.G.Q. 2001. Adsorption kinetics for the removal of chromium(VI) from aqueous solution by adsorbents derived from used tyres and sawdust. Chemical Engineering Journal, 84: 95-105.

Kadirvelu, K; Thamaraiselvi, K; Namasivayam, C. 2001. Adsorption of nickel(II) from aqueous solution onto activated carbon prepared from coirpith. Sep. Pur. Technol, 24: 497-505.

Karthikeyan, T.; Rajgopal, S,; Miranda, L.R. 2005. Chromium (VI) adsorption from aqueous solution by Hevea brasilinesis sawdust activated carbon. Journal of hazardous materials, 124: 192-199;

Liu, S.X.; Chen, X.; Liu, Z.F.; Wang, H.L. 2007. Activated carbon with excellent chromium(VI) adsorption performance prepared by acid-base surface modification. Journal of Hazardous Materials, 141: 315-319.

Mohana, M.; Pittman Jr, C.U. 2006. Activated carbons and low cost adsorbents for remediation of tri- and hexavalent chromium from water. Journal of Hazardous Materials, 137: 762-811.

Mohanty, K.; Jha, M.; Meikap, B.C.; Biswas, M.N. 2005. Removal of chromium (VI) from dilute aqueous solutions by activated carbon developed from Terminalia arjuna nuts activated with zinc chloride. Chemical Engineering Science, 60: 3049-3059.
Natale, F. D.; Lancia, A.; Molino, A.; Musmarra, D. 2007. Removal of chromium ions form aqueous solutions by adsorption on activated carbon and char. Journal of Hazardous Materials, 145: 381-390.

Ozacar, M. 2003. Equilibrium and Kinetic Modelling of Adsorption of Phosphorus on Calcined Alunite. Adsorption, 9: 125-132.

Schneider, R.M.; Cavalin, C.F.; Barros, M.A.S.D.; Tavares, C.R.G. 2007. Adsorption of chromium ions in activated carbon. Chemical Engineering Journal, 132: 355-362

Selvi, K.; Pattabi, S.; Kadirvelu, K. 2001. Removal of Cr(VI) from aqueous solution by adsorption onto activated carbon. Biores. Technol, 80: 87-89.

Souza, R.S. Utilização de técnicas de planejamento fatorial estatístico na otimização de variáveis de processo de adsorção de cromo VI em CAG. Anais do $10^{\circ}$ Encontro de profissionais da química da Amazônia - Recursos Naturais: uma reflexão para os profissionais da Química.2007.

Zhao, N.; Wei, N.; Li, J.; Qiao, Z.; Cui, J.; He, F. 2005. Surface properties of chemicallymodified activated carbons for adsorption rate of Cr (VI). Chemical Engineering Journal, 115: 133-138.

Recebido em 26/08/2008

Aceito em 18/11/2008 
